\title{
Physical Reality, Theoretical Physics, and Mathematics-15 Years Later
}

\author{
Piret Kuusk \\ Institute of Physics, University of Tartu \\ W. Ostwaldi 1, \\ Tartu 50411, Estonia \\ E-mail:Piret.Kuusk@ut.ee
}

\begin{abstract}
Physicists usually admit a rather naive concept of physical reality: it is the object they are investigating using experiments understood as actions in the real world. In theoretical physics, the object of investigation is not the real world itself but its mathematical description which is considered as describing physical reality as exactly as possible. I present arguments from philosophy of science and from recent work in the theory of gravitational physics which indicate a possibility to defend also antirealistic views, and clarify in which sense both standpoints can be viable.
\end{abstract}

Keywords: general relativity, scientific antirealism, scientific realism, spacetime, teleparallel equivalent of general relativity

\section{Introduction}

The starting point of the current paper is a book which was published 15 years ago-Estonian Studies in the History and Philosophy of Science, edited by Rein Vihalemm (2001). The book consists of 22 papers by 24 authors, among them philosophers and historians as well as working scientists. I am a physicist, working in fundamental theoretical physics already for a long time. Under the strong encouragement of the Professor of Philosophy of Science at the University of Tartu, Rein Vihalemm, I prepared a paper for the abovementioned book on relations between physics and mathematics from the viewpoint of a working theoretical physicist (Kuusk, 2001). Unfortunately there was a strict page limit and so my paper was published without the last section, the summary. In the current paper, I proceed from this summary, which reflects a rather naive concept 
of physical reality, often held by working physicists, and indicate how recent developments in theoretical physics strongly suggest to move from favouring naive realism to sympathizing also with several versions of antirealism, discussed in the philosophy of science.

My approach to the question about realism and antirealism in physics is inspired by Maddy's view on naturalism in mathematics (Maddy, 1997, pp. 183-205). She proposed to consider the actual work of mathematicians and claimed that this can be done using methods of science, for example, the hypotheticodeductive method, although mathematics itself is different from science and, in their professional work, mathematicians use axiomatic methods and strict logic instead of methods of science. But according to Maddy, doing mathematics is a scientific activity which can be naturalized. My strategy is analogous: I proceed from the actual developments in fundamental theoretical physics and investigate how scientists themselves interpret and understand their work, keeping an eye on the problem of realism and antirealism.

\section{Physical reality: a received view of working physicists}

Many working physicists surely will agree with the following statements which in fact were expressed, although not published, in my paper (Kuusk, 2001):

1. Physical investigations are a natural continuation of everyday commonsense experience outside the range of direct sensory perception.

2. In classical physics, the definition of physical reality is modelled as a continuation of the directly perceivable everyday world. However, even in the framework of classical physics, physical research does not concern everything in the world but only certain phenomena which physics is able to investigate. These phenomena are discovered by the investigator and not created by him.

3. The generally accepted and experimentally verified part of the description of the quantum physical world is given in the language of mathematics. This means that a definitely adequate way of thinking about it is in mathematical formulas. But just as everyday experience is not described in sensations and perceptions but in a (natural) language involving concepts and sentences, quantum physical reality tends to be described analogously by means of concepts and propositions. These are formed on the basis of mathematical 
notions and formulas but do not coincide with them. They contain human imagination which fills the gaps of the formal structure given by mathematics.

4. Two types of mathematical reasoning can be distinguished in the practice of theoretical physics - the phenomenological approach and the mathematical approach. From the standpoint of the phenomenological approach, essential parts of mathematics can be considered as a description of physical reality, that is, mathematics is deducible from physics. From the standpoint of the mathematical approach, ontological consequences about the physical reality can be derived from at least some mathematical theories - that is, physics is deducible from mathematics. Both of these approaches have been sufficiently successful, and we have no reason to prefer one of them and reject the other.

5. At present the ultimate aim of at least fundamental theoretical physics is believed to be the unification of physics. Its driving force seems to be the mathematical language and not experimental results.

\section{Realistic stance of the received view}

This summary of the received view of working physicists should not be considered as a philosophical statement, but as a reflection of scientists about their professional work. It can be taken as expressing a philosophical stance, described by van Fraassen:

A philosophical position can consist in a stance (attitude, commitment, approach, a cluster of such — possibly including some propositional attitudes such as beliefs as well). Such a stance can of course be expressed, and may involve or presuppose some beliefs as well, but cannot be simply equated with having beliefs or making assertions about what there is. (van Fraassen, 2002, pp. 47-48)

The stance of the received view of physicists clearly favours realism in respect of the physical world. It proceeds from the commonsense understanding of external world as really existing and extends the real existence to all physical processes. In particular, it states that physical phenomena are discovered and not created by the investigator. However, it also permits deducing ontological consequences about the physical reality from mathematics, which still introduces a possibility to infer properties of physical objects from their theoretical and mathematical descriptions. 


\section{Arguments for antirealism from philosophy of science}

In the 1920s-1930s, the leading movements in the philosophy of science were logical empiricism and logical positivism, which based science exclusively on human experience and logical inferences and did not care about the ontology of the real world. A profound critisism by Quine (1951) undermined their credibility. In the 1950s-1960s, scientific realism returned and prevailed in the philosophy of science, as well as in the received view of scientists.

However, in the everyday work of physicists, philosophers soon began to notice also several antirealistic standpoints.

Nancy Cartwright aims to be as close to actual physics as possible, and she claims to be an empiricist, since physics is an empirical science. She has published two interesting books about physical laws: How the Laws of Physics Lie (Cartwright, 1983) where she defends realism with respect to unobservable theoretical entities, but antirealism with respect to general theories, and The Dappled World: A Study of the Boundaries of Science (Cartwright, 1999), where she defends realism also with respect to theories and expressed a pluralistic ("dappled") view on scientific lawfulness. Her main argument in the first book, with a title that sounds somewhat insulting for physicists, is that great principles of the $19^{\text {th }}$ century physics - conservation of energy, increase of entropy, conservation of electric charge, etc.-hold only ceteris paribus, but in actual experiments this condition is never fulfilled. Everything that is going on in the world is idiosyncratic, so at best only phenomenological laws can describe natural processes. In the second book, she changed her mind and considered also general laws to be true, still not unconditionally true, but true as a patchwork. In some sense this means plurality in the lawfulness of the world.

Bas van Fraassen (1980) based his profoundly antirealistic constructive empiricism on Sellars's $(1956 ; 1963)$ concept of two frameworks for representing the world: the manifest image, inherited from everyday life and earlier philosophy, and the scientific image, which is based on postulates established by our best contemporary science. Since both of these frameworks are logically closed but partly contradictory, they cannot be valid simultaneously. Sellars was a scientific realist and considered both frameworks to be appropriate for describing the real world. However, van Fraassen made a different choice: he retained the scientific image and did not consider the 'real world' at all. In his influential book The Scientific Image (van Fraassen, 1980) he wrote: 
Science aims to give us theories which are empirically adequate; the acceptance of a theory involves as belief that it is empirically adequate (van Fraassen, 1980, p. 12),

and

To present a theory is to specify a family of structures, its models; and secondly, to specify certain parts of those models (the empirical substructures) as candidates for the direct representation of observable phenomena (van Fraassen, 1980, p. 64).

According to his constructive empiricism, the aim of science is not finding a true description of the real world, in the sense of the correspondence theory of truth, but a description which is true with respect to empirical data only.

Ronald Giere developed the idea of scientific models as the basis of science, but took into account also the autor of the models, together with her intentions to use them and to which purpose:

I argue for an intentional conception of representation in science that requires bringing scientific agents and their intentions into the picture. (Giere, 2009, p. 269).

How does one connect abstract models to specific real physical systems? This requires at least two processes which I call 'interpretation' and 'identification' (Giere, 1988, pp. 74-76).

For interpretation, elements of an abstract principled model are provided with general physical interpretations such as 'mass', 'position', and 'velocity'. Such interpretations are already present in the statements that characterize the principled models. Scientists do not begin with an "uninterpreted" formalism and then "add" interpretations. For identification, elements of a representational model are identified (or coordinated) with elements of a real system. Do we, as theorists of science, need to give a more detailed account of the processes of interpretation and identification? I think not. (Giere, 2009, p. 271)

Since the question of correspondence of elements of models to elements of the real world is declared to be unimportant, it is clear that Giere's concept of science is antirealistic. 
Physicists usually do not read philosophical papers and are not interested in discussions of philosophers. However, sometimes their own professional work leads them to standpoints which have counterparts in contemporary philosophy. In what follows I present a recent example from gravitational physics which seems to pose a challenge to scientific realism.

\section{An argument for antirealism from gravitational physics}

History of physics shows that not a single physical theory can live forever. We know that about 700 years ago, physics of Aristotle was considered to be the true description of the real world, and at present we cannot even imagine which theories will replace, for example, quantum mechanics or Einstein's general relativity after another 700 years. This can be considered as an argument against scientific realism.

But intuition and imagination certainly have a significant heuristic role in the scientific work, and this inspires physicists to interpret their theories more or less realistically, at least in the framework of their everyday scientific investigations. They tend to keep their realistic stance even in situations where the notion of physical reality seems to be ambiguous. This can be illustrated by the following example from recent developments in gravitational physics.

At present, general relativity (GR) is widely accepted as the most reliable description of gravitational phenomena and it is verified through a myriad of experiments and observations. According to GR, gravitational phenomena are direct consequences of the fact that spacetime is curved in a specific way which is determined by the matter content of spacetime. An archetypal example of a 2-dimensional curved manifold is the surface of a sphere, as distinct from a flat 2-dimensional manifold of a plane. Although spacetime is 4-dimensional, there are numerous pictorial illustrations for explaining the mathematical results following from equations of GR and their solutions, interpreted as manifestations of spacetime curvature; the most famous of them appear in connection with black holes and big bang cosmology. Such illustrations are extremely common in popular science books, but are often used also in scientific publications.

Mathematical formulation of GR includes a claim that the spacetime manifold is curved (it may be flat as a special case, if gravitation is ignored), but has no torsion. Curvature and torsion are two independent characteristics of a 
differentiable manifold, their precise mathematical definitions are presented in basic differential geometry. An intuitive picture of a 2-dimensional manifold with torsion and without curvature is given by a winding staircase.

In quest of a unified theory for gravitation and electromagnetism, Einstein proposed to model spacetime as a manifold with torsion, but without curvature (Einstein, 1928a; 1928b). He derived the corresponding field equations and tried to find their proper interpretation. But his attempt failed, since the theory did not allow a solution for an isolated spherically symmetric static material body which could describe, for example, familiar gravitational properties of the Sun or the Earth.

The theory was abandoned and forgotten for a long time, but revived in recent years in a slightly different form. It was noticed at once that with certain mathematical redefinitions and manipulations, equations of the new formulation coincide with those of GR. From this it was deduced that the theory, dubbed 'Teleparallel Equivalent of General Relativity' or TEGR (Aldrovandi \& Pereira, 2013), can be considered as an alternative description of gravitational phenomena. Although matematically equivalent, it is conceptually very different from GR. In particular, the physical spacetime is considered to be not a curved torsionless manifold as in GR, but a flat manifold with torsion.

Since we all agree that we live in space and time, and that in a scientific description space and time can be considered as parts of a 4-dimensional spacetime, it is reasonable to ask: Which in fact is the geometry of real, physical spacetime? Is it curved and torsionless, as it is assumed in GR, or is it flat and with torsion, as it is assumed in TEGR? The answer cannot be deduced from experiments or observations, since both theories are mathematically equivalent and differ only in interpretations. Therefore we can always establish correspondences between their observational consequences. In a sense, physical spacetime turns out to be unobservable: we can investigate it in the framework of either one or the other theory, but we can never find out which of these two theories with noncompatible basic assumptions is a true description of the real spacetime. The notion of real spacetime seems to become superfluous, although we clearly can locally observe and measure spatial lengths and intervals of time, and scientists certainly continue to illustrate their results with figures which follow from the mathematical model they choose to represent spacetime in their investigations.

But this is not the end of the story. There are hints that existing theories of gravity may need modifications to conform to all cosmological observations. It 
turns out that although GR and TEGR are mathematically and observationally equivalent, their analogously modified extensions are not. So there seems to appear a possibility to find from observations, which modified theory is in the best correspondence with observations, and from its origin, GR or TEGR, determine the basic characteristics of spacetime, curvature and/or torsion. But as we already mentioned, GR is up to now in a good agreement with observations and experiments within the solar system, and gives a more or less satisfactory large scale description of the universe. So modifications of the underlying theory of gravitation can introduce only small corrections into the description of gravitational phenomena occurring here and now. Our cosmological observations are scarce, it is difficult to obtain really new cosmological data, and usually they have significant statistical and systematic errors. It is possible that in the foreseeable future, new observational data allow interpretations within both modified theories of gravity - that is, modified GR as well as modified TEGR. This means that we must consider our mathematical models of spacetime as ultimate knowledge about the structure of the universe and conform our intuitions about it to the model we have chosen to be the most appropriate, admitting that the nearest model which possibly also can explain the observations may be conceptually very different, as are GR and TEGR. As to the unforeseeable future, we are always haunted by a possibility that there will be a totally new and different theory for describing gravitational phenomena in our universe.

\section{Concluding remarks}

Fifteen years ago I summarized my views about the theoretical physics and mathematics, and presented them as a received view of working physicists. At present I dare not formulate beyond my personal understanding.

It seems that contemporary fundamental physics aims to find a (mathematically formulated) story that conforms to experimental and observational data as much and as exactly as possible. It is surely only a mathematical model, but I am also very much disposed to consider it as a more or less exact description of physical reality. However, especially in the regions which are far from being directly perceivable, it is possible to build models which look more like mathematical fantasies than plausible descriptions of reality. Indeed, in such regions experimental and observational data are scarce, leaving many very different possibilities for binding 
them together into a unified mathematical framework. Now the investigator is facing a dilemma: she can uphold a realistic standpoint in respect of a specific model she is just investigating, or she can take a broader point of view and prefer an antirealistic standpoint in respect of all possible models. Each of these standpoints has a justification, but of course they must be applied in appropriate frameworks and not simultaneously.

\section{Acknowledgement}

I want to thank Professor Paul Hoyningen-Huene for calling my attention to his interesting lecture 'Are there good arguments against scientific realism?' (Hoyningen-Huene, 2016), which gave me new insights into my problems.

\section{References}

Aldrovandi, R. \& Pereira, J. G. (2013), Teleparallel Gravity: An Introduction, Dordrecht, Heidelberg, New York \& London: Springer. https://doi.org/10.1007/978-94-007-5143-9

Cartwright, N. (1983), How the Laws of Physics Lie, Oxford: Oxford University Press. https://doi.org/10.1093/0198247044.001.0001

- (1999), The Dappled World: A Study of the Boundaries of Science, Cambridge: Cambridge University Press. https://doi.org/10.1017/CBO9781139167093

Einstein, A. (1928a), 'Riemann-Geometrie mit Aufrechthaltung des Begriffes des Fernparallelismus,' in Sitzungsberichte der Preussischen Akademie der Wissenschaften, Physikalisch-Mathematische Klasse, Berlin: Deutsche Akademie der Wissenschaften zu Berlin, pp. 217-221.

(1928b), 'Neue Möglichkeit für eine einheitliche Feldtheorie von Gravitation und Elektricität,' in Sitzungsberichte der Preussischen Akademie der Wissenschaften, Physikalisch-Mathematische Klasse, Berlin: Deutsche Akademie der Wissenschaften zu Berlin, pp. 224-227.

Giere, R. (2009), 'An agent-based conception of models and scientific representation,' Synthese, vol. 172, no. 2, pp. 269-281. https://doi.org/10.1007/s11229-009-9506-z

Hoyningen-Huene, P. (2016), 'Are there good arguments against scientific realism?' Invited lecture at the conference of the Gesellschaft für Wissenschaftsphilosophie GWP on 10 March 2016 at Düsseldorf University. Retrieved from https://www. youtube.com/user/PHoyningen [accessed Dec 2016] 
Kuusk, P. (2001), 'Physical reality, theoretical physics, and mathematics,' in R. Vihalemm (ed.) Estonian Studies in the History and Philosophy of Science, Boston Studies in the Philosophy of Science, Dordrecht, Boston \& London: Cluwer Academic Publishers (Springer Netherlands), vol. 219, pp. 204-214. (Softcover reprint, 2014) https://doi.org/10.1007/978-94-010-0672-9_15

Maddy, P. (1997), Naturalism in Mathematics, Oxford: Oxford University Press.

Quine, W. v. O. (1951), 'Two dogmas of empiricism,' The Philosophical Review, vol. 60, pp. 20-43. https://doi.org/10.2307/2181906

Sellars, W. (1956), 'Empiricism and the philosophy of mind,' in H. Feigl \& M. Scriven (eds.) Minnesota Studies in the Philosophy of Science, Volume I: The Foundations of Science and the Concepts of Psychology and Psychoanalysis, Minneapolis: University of Minnesota Press, pp. 253-329.

(1963), 'Philosophy and the scientific image of man,' in Science, Perception, and Reality, New York: Humanities Press, pp. 1-40.

van Fraassen, B. (1980), The Scientific Image, Oxford: Clarendon Press. https://doi.org/10.1093/0198244274.001.0001

(2002), The Empirical Stance, New Haven \& London: Yale University Press.

Vihalemm, R., ed. (2001), Estonian Studies in the History and Philosophy of Science, Boston Studies in the Philosophy of Science, Dordrecht, Boston \& London: Cluwer Academic Publishers (Springer Netherlands), vol. 219. (Softcover reprint, 2014)

Piret Kuusk is senior research scientist and head of the Laboratory of Theoretical Physics at the Institute of Physics, University of Tartu. She obtained doctoral degree in Theoretical and Mathematical Physics in 1990. Her research is part of fundamental theoretical physics and is mainly focused on gravitational phenomena. 